PROCEEDINGS OF THE

AMERICAN MATHEMATICAL SOCIETY

Volume 133, Number 8, Pages 2439-2447

S 0002-9939(05)08137-2

Article electronically published on March 21, 2005

\title{
THE MARGULIS INVARIANT FOR PARABOLIC TRANSFORMATIONS
}

\author{
VIRGINIE CHARETTE AND TODD A. DRUMM
}

(Communicated by Wolfgang Ziller)

\begin{abstract}
In this note, we extend the definition of Margulis' signed Lorentzian displacement to parabolic transformations in $S O(2,1) \ltimes \mathbb{R}^{2,1}$. We show that the standard propositions about the "sign" of the transformations all hold true for parabolic elements also. In particular, we show that Margulis' opposite sign lemma holds.
\end{abstract}

\section{INTRODUCTION}

Margulis [5, 6] proved the existence of free groups of affine transformations that act properly discontinuously on $\mathbb{R}^{3}$. This surprising discovery answered in the negative a question posed by Milnor [8]: Is an affine group that acts properly discontinuously on $\mathbb{R}^{n}$ virtually solvable?

Fried and Goldman [4] show that if an affine group acting properly on $\mathbb{R}^{3}$ is not virtually solvable, its linear part must preserve a metric of signature $(2,1)$. On the other hand, Mess [7] shows that the fundamental group of a closed surface cannot act properly discontinuously on $\mathbb{R}^{3}$. Thus the only remaining possibilities are free groups generated by hyperbolic or parabolic transformations.

Margulis' counterexamples are free, discrete groups whose linear parts are purely hyperbolic. He suggested that one could not get a free group containing parabolics to act properly discontinuously on $\mathbb{R}^{3}$, unless of course it is cyclic. However, as is shown in [1, any free, discrete group of isometries of the hyperbolic plane admits an affine deformation that acts properly discontinuously on $\mathbb{R}^{3}$, preserving an affine $(2+1)$-spacetime structure.

These findings were made possible by engineering fundamental domains for these actions, bounded by objects called crooked planes, which are studied extensively in, for example, 3. This approach diverges from Margulis' original study of the signed Lorentzian displacement associated to an affine hyperbolic transformation, also referred to in the literature as the Margulis invariant.

Here we extend the Margulis invariant to parabolic transformations and generalize the ground breaking results of Margulis to groups which contain these transformations. This effort may be viewed in the context of an ongoing program to better understand these non-amenable actions; in particular, we aim to understand the relationship between the existence of crooked fundamental domains and the behavior of the Margulis invariant in a group.

Received by the editors February 14, 2003.

2000 Mathematics Subject Classification. Primary 53A15; Secondary 83A05. 
Section 2 presents basic definitions and a brief survey of Margulis' work, focusing on those results we will generalize. In Section 3 we extend the Margulis invariant to parabolic transformations. In Section 4, we generalize the results given in Section2 using the extended Margulis invariant. In particular, we show that if the signs of the new invariant for a hyperbolic and parabolic transformation are opposite, then the group they generate cannot possibly act properly discontinuously on $\mathbb{R}^{2,1}$.

\section{BACKGROUND}

2.1. Geometry. Let $\mathbb{A}^{2,1}$ denote a three-dimensional affine space with the following additional structure. Its associated vector space of directions

$$
\mathbb{R}^{2,1}=\left\{p-q \mid p, q \in \mathbb{A}^{2,1}\right\}
$$

which is isomorphic to $\mathbb{R}^{3}$ as a vector space, is endowed with the standard Lorentzian scalar product

$$
\mathbb{B}(\mathrm{x}, \mathrm{y})=x_{1} y_{1}+x_{2} y_{2}-x_{3} y_{3},
$$

where $\mathbf{x}=\left[\begin{array}{lll}x_{1} & x_{2} & x_{3}\end{array}\right]^{T}, \mathbf{y}=\left[\begin{array}{lll}y_{1} & y_{2} & y_{3}\end{array}\right]^{T} \in \mathbb{R}^{3}$. Thus, $\mathbb{A}^{2,1}$ is a Minkowski $(2+1)$ spacetime.

A non-zero vector $\mathrm{x}$ is said to be null (resp. timelike, spacelike) if $\mathbb{B}(\mathrm{x}, \mathrm{x})=0$ (resp. $\mathbb{B}(x, x)<0, \mathbb{B}(x, x)>0)$. A null vector is future-pointing if its third coordinate is positive - this corresponds to choosing a connected component of the set of timelike vectors, or a time-orientation.

The Lorenzian cross-product is the unique bilinear map

$$
\otimes: \mathbb{R}^{2,1} \times \mathbb{R}^{2,1} \longrightarrow \mathbb{R}^{2,1}
$$

such that $\mathbb{B}(\mathrm{u}, \mathrm{v} \otimes \mathrm{w})=\operatorname{Det}\left(I_{2,1}[\mathrm{u} \vee \mathrm{w}]\right)$, where $I_{2,1}$ is the Gramm matrix for the standard Lorentzian scalar product, relative to the standard basis

$$
I_{2,1}=\left[\begin{array}{ccc}
1 & 0 & 0 \\
0 & 1 & 0 \\
0 & 0 & -1
\end{array}\right] \text {. }
$$

Let $\operatorname{Aff}\left(\mathbb{A}^{2,1}\right)$ denote the group of all affine transformations that preserve the Lorentzian scalar product on the space of directions; $\operatorname{Aff}\left(\mathbb{A}^{2,1}\right)$ is isomorphic to $\mathrm{O}(2,1) \ltimes \mathbb{R}^{2,1}$. We shall restrict our attention to those transformations whose linear parts are in $\mathrm{SO}(2,1)^{0}$, thus preserving orientation and time orientation.

Denote projection onto the linear part of an affine transformation by

$$
\mathbb{L}: \operatorname{Aff}\left(\mathbb{A}^{2,1}\right) \rightarrow \mathrm{O}(2,1) .
$$

The isomorphism between $\mathrm{SO}(2,1)^{0}$ and $\operatorname{Isom}\left(\mathbb{H}^{2}\right)$ gives rise to the following terminology.

Definition 2.1. Let $g \in \mathrm{SO}(2,1)^{0}$ be a nonidentity element. Then

- $g$ is hyperbolic if it has three, distinct real eigenvalues;

- $g$ is parabolic if its only eigenvalue is 1 .

We also call $\gamma \in \operatorname{Aff}\left(\mathbb{A}^{2,1}\right)$ hyperbolic (resp. parabolic) if its linear part $\mathbb{L}(\gamma)$ is hyperbolic (resp. parabolic).

Note that we do not consider pure translations to be either hyperbolic or parabolic, since their linear part is the identity.

The set of hyperbolic transformations in $\operatorname{Aff}\left(\mathbb{A}^{2,1}\right)$ will be denoted by $\mathcal{H}$ and the set of parabolic transformations by $\mathcal{P}$. 
2.2. Results for hyperbolic transformations. Suppose $g \in \mathrm{SO}(2,1)^{0}$ is hyperbolic; its eigenvalues are $\lambda, 1,1 / \lambda$, for some $0<\lambda<1$. Associated to $g$ is a null frame

$$
\left\{\mathrm{x}^{0}(g), \mathrm{x}^{-}(g), \mathrm{x}^{+}(g)\right\}
$$

where $\mathbf{x}^{-}(g)$ (resp. $\mathbf{x}^{+}(g)$ ) is a null, future-pointing $\lambda$-eigenvector (resp. a $1 / \lambda$ eigenvector), chosen to be of unit Euclidean length, and $x^{0}(g)$ is the unique 1eigenvector such that $\mathbb{B}\left(\mathrm{x}^{0}(g), \mathrm{x}^{0}(g)\right)=1$ and $\left\{\mathrm{x}^{0}(g), \mathrm{x}^{-}(g), \mathrm{x}^{+}(g)\right\}$ is positively oriented. If $\gamma \in \mathcal{H}$, we write

$$
\left\{x^{0}(\gamma), x^{-}(\gamma), x^{+}(\gamma)\right\}:=\left\{x^{0}(\mathbb{L}(\gamma)), x^{-}(\mathbb{L}(\gamma)), x^{+}(\mathbb{L}(\gamma))\right\}
$$

Every hyperbolic $\gamma$ admits a $\gamma$-invariant line $C_{\gamma}$ which is parallel to $\mathrm{x}^{0}(\gamma)$. The transformation $\gamma$ acts by translation on $C_{\gamma}$. On the subset $\mathcal{H} \subset \operatorname{Aff}\left(\mathbb{A}^{2,1}\right)$ of hyperbolic elements, we define the signed Lorentzian displacement of $\gamma$ to be the function

$$
\alpha: \mathcal{H} \rightarrow \mathbb{R}
$$

such that

$$
\alpha(\gamma)=\mathbb{B}\left(\gamma(x)-x, x^{0}(\gamma)\right)
$$

for any $x \in C_{\gamma}$.

The action on $C_{\gamma}$ is given by

$$
\gamma(x)=x+\alpha(\gamma) \mathrm{x}^{0}(\gamma)
$$

for every $x \in C_{\gamma}$.

The proof of the next lemma is straightforward and, furthermore, analogous to that of Lemma 3.2 ,

Lemma 2.2. Suppose $\gamma \in \mathcal{H}$. Then

(1) for any $x \in \mathbb{A}^{2,1}, \mathbb{B}\left(\gamma(x)-x, \mathrm{x}^{0}(\gamma)\right)=\alpha(\gamma)$;

(2) $\alpha(\gamma) \neq 0$ if and only if $\gamma$ acts freely;

(3) for any $\eta \in \operatorname{Aff}\left(\mathbb{A}^{2,1}\right), \alpha\left(\eta \gamma \eta^{-1}\right)=\alpha(\gamma)$;

(4) for any $\gamma \in \operatorname{Aff}\left(\mathbb{A}^{2,1}\right), \alpha\left(\gamma^{n}\right)=|n| \alpha(\gamma)$.

Any two hyperbolic elements in $\mathrm{SO}(2,1)^{0}$ with the same eigenvalues, or trace, are conjugate. Furthermore, it can be shown that any two hyperbolic elements in $\operatorname{Aff}\left(\mathbb{A}^{2,1}\right)$ with the same linear part and signed Lorentzian displacement are conjugate by a translation. Thus, the trace of the linear part and the value of $\alpha$ determine the conjugacy classes of hyperbolic elements in $\operatorname{Aff}\left(\mathbb{A}^{2,1}\right)$.

Since $\mathrm{x}^{0}\left(\gamma^{-1}\right)=-\mathrm{x}^{0}(\gamma)$, we have that $\alpha\left(\gamma^{-1}\right)=\alpha(\gamma)$. When $\gamma$ acts freely, the sign of $\alpha(\gamma)$ indicates the direction in which $\gamma$ displaces points.

In order to investigate properness of actions of discrete groups in $\operatorname{Aff}\left(\mathbb{A}^{2,1}\right)$, it is important to understand the topology of $\mathbb{A}^{2,1}$. However, the Lorentzian scalar product does not correspond to the underlying topology. The Euclidean metric, though not $\mathrm{SO}(2,1)^{0}$-invariant, does define the underlying topology. The Lorentzian scalar product is connected to the usual $\mathbb{R}^{3}$ metric, and thus the standard topology, via the following definition.

Definition 2.3. A hyperbolic linear transformation $g$ is $\epsilon$-hyperbolic if

$$
\rho\left(\mathrm{x}^{+}(g), \mathrm{x}^{-}(g)\right)>\epsilon,
$$

where $\rho$ denotes Euclidean distance. 
A simple calculation gives us the following lemma.

Lemma 2.4. If $g \in \mathrm{SO}(2,1)^{0}$ is $\epsilon$-hyperbolic, then

$$
\left\|\mathrm{x}^{0}(g)\right\|<\frac{4}{\epsilon}-1
$$

where \|\| denotes Euclidean length. In particular, for every $\epsilon>0$, the set of all unit spacelike vectors $\mathrm{x} \in \mathbb{A}^{2,1}$ such that $\mathrm{x}=\mathrm{x}^{0}(g)$ for some $\epsilon$-hyperbolic $g$, is compact.

We close this section by stating Margulis' sign condition on $\alpha$ for proper actions.

Lemma 2.5 (Margulis [5, 6]; see also Drumm [2]). Let $\gamma_{1}, \gamma_{2} \in \mathcal{H}$ and suppose $\alpha\left(\gamma_{1}\right) \alpha\left(\gamma_{2}\right)<0$. Then the group generated by $\gamma_{1}$ and $\gamma_{2}$ cannot act properly discontinuously on $\mathbb{A}^{2,1}$.

\section{A NON-NORMALIZED MARGULIS INVARIANT}

We now wish to extend the definition of the signed Lorentzian displacement (1) to parabolic transformations. There are many difficulties in extending this definition, two of which stem from the way one defines $x^{0}(g)$.

First, the direction of $x^{0}(g)$ depends on the null eigenvectors, which correspond to the real eigenvalues not equal to one. But only hyperbolic elements of $\mathrm{SO}(2,1)^{0}$ have three real eigenvectors.

We can get around this difficulty by noticing that if $x \in \mathbb{R}^{2,1}$ is a null or timelike vector which is not an eigenvector of a hyperbolic $g$, then $\left\{\mathrm{x}^{0}(g), \mathrm{x}, g \mathrm{x}\right\}$ is also a positively-oriented basis for $\mathbb{R}^{2,1}$. This observation extends to parabolic transformations:

Definition 3.1. Let $g$ be a linear transformation that is either hyperbolic or parabolic. A 1-eigenvector $\mathrm{v}$ is said to be positive relative to $g$ if $\{\mathrm{v}, \mathrm{x}, g \times\}$ is a positively oriented basis, where $\mathrm{x}$ is any null or timelike vector which is not an eigenvector of $g$.

Note that $\mathrm{v}$ is positive relative to $g$ if and only if $-\mathrm{v}$ is positive relative to $g^{-1}$. Also, the direction of positive vectors varies continuously, for $g \in \mathcal{H} \cup \mathcal{P}$.

The second difficulty appears in the choice of the Lorentzian length of $x^{0}(g)$. The fixed eigenvector for a parabolic element of $\mathrm{SO}(2,1)^{0}$ is a null vector, so we cannot force the Lorentzian length of a fixed vector to be 1 . Therefore our "new displacement" cannot be normalized according to the length of a fixed vector of $g$. Instead, the image will be a linear function rather than a number.

Let $F(g)$ be the oriented one-dimensional space of fixed eigenvectors of $g$. The orientation of $F(g)$ is defined above. Given an element $\gamma \in \mathcal{H} \cup \mathcal{P}$ with $\mathbb{L}(\gamma)=g$, we consider the linear function on $F(g)$ :

$$
\tilde{\alpha}(\gamma)=\mathbb{B}(\gamma(x)-x, \cdot),
$$

where $x$ is an arbitrary point in $\mathbb{A}^{2,1}$.

When $\gamma$ is hyperbolic, this non-normalized Margulis invariant $\tilde{\alpha}$ corresponds to $\alpha$ in the following simple manner:

$$
\alpha(\gamma)=\tilde{\alpha}(\gamma)\left(x^{0}(g)\right) .
$$

However, before accepting this definition as our new Margulis invariant, we will first show that $\tilde{\alpha}$ acts just like $\alpha$ for parabolic, as well as hyperbolic, elements. 
Lemma 3.2. Suppose $\gamma \in \mathcal{H} \cup \mathcal{P}$. Then

(1) the definition of the function $\tilde{\alpha}(\gamma)=\mathbb{B}(\gamma(x)-x, \cdot)$ is independent of the choice of $x \in \mathbb{A}^{2,1}$

(2) $\tilde{\alpha}(\gamma)$ is identically zero if and only if $\gamma$ has a fixed point;

(3) for any $\eta \in \operatorname{Aff}\left(\mathbb{A}^{2,1}\right)$, $\tilde{\alpha}\left(\eta \gamma \eta^{-1}\right)(h(\mathrm{v}))=\tilde{\alpha}(\gamma)(\mathrm{v})$, where $\mathrm{v} \in F(g)$ and $h=\mathbb{L}(\eta)$

(4) for any $n \in \mathbb{Z}$ and $\mathrm{v} \in F(g)$, $\tilde{\alpha}\left(\gamma^{n}\right)(\mathrm{v})=n \tilde{\alpha}(\gamma)(\mathrm{v})$.

Proof. For a parabolic element $p \in \mathrm{SO}(2,1)^{0}$ we can choose a basis $\{\mathrm{v}, \mathrm{w}, \mathrm{u}\}$ for $\mathbb{R}^{2,1}$ such that $\mathrm{v} \in F(p), \mathbb{B}(\mathrm{v}, \mathrm{w})=0$ and $\mathbb{B}(\mathrm{v}, \mathrm{u}) \neq 0$. The matrix of $p$ relative to this basis is of the form

$$
\left[\begin{array}{lll}
1 & a & b \\
0 & 1 & c \\
0 & 0 & 1
\end{array}\right],
$$

with $a, c \neq 0$. Thus, if $g \in \mathrm{SO}(2,1)^{0}$ is either parabolic or hyperbolic, the range of $g-I d$ is precisely the Lorentz-perpendicular plane of the eigenspace $F(g)$.

Let $g=\mathbb{L}(\gamma)$. Choose $x_{o} \in \mathbb{A}^{2,1}$ and set

$$
\mathrm{v}_{\gamma}=\gamma\left(x_{o}\right)-x_{o}
$$

We can think of $\mathrm{v}_{\gamma}$ as being the translational part of $\gamma$, when $x_{o}$ acts as the origin.

Let $y \in \mathbb{A}^{2,1}$. Then

$$
\gamma(y)-y-(\gamma(x)-x)=(g-I d)(y-x) .
$$

Since the range of $g-I d$ is $\mathrm{v}^{\perp}$, for $\vee \in F(g)$,

$$
\mathbb{B}(\gamma(x)-x, \mathrm{v})=\mathbb{B}(\gamma(y)-y, \mathrm{v}),
$$

proving statement (11). In particular,

$$
\tilde{\alpha}(\gamma)=\mathbb{B}\left(\mathrm{v}_{\gamma}, \cdot\right)
$$

For statement (2), suppose that $\gamma$ has a fixed point. Then choosing $x$ in (2) to be that fixed point, we obtain that the function $\tilde{\alpha}(\gamma)$ is identically zero.

Conversely, suppose that $\tilde{\alpha}(\gamma)=0$. Then $\mathrm{v}_{\gamma} \in \mathrm{v}^{\perp}$, for any 1-eigenvector $\mathrm{v}$ of $g$. Thus the following matrix equation has a solution:

$$
(g-I d) \mathrm{x}=-\mathrm{v}_{\gamma},
$$

which means that $x_{o}+\mathrm{x}$ is a fixed point.

To prove statement (3), let $h=\mathbb{L}(\eta)$ be the linear part of $\eta$. If $\vee \in F(g)$, then $h(\mathrm{v})$ is a fixed point for $h g h^{-1}$. Choose an arbitrary $x \in \mathbb{A}^{2,1}$. Then

$$
\begin{aligned}
\tilde{\alpha}\left(\eta \gamma \eta^{-1}\right)(h(\mathrm{v})) & =\mathbb{B}\left(\eta \gamma \eta^{-1}(x)-x, h(\mathrm{v})\right) \\
& =\mathbb{B}\left(h\left(\gamma \eta^{-1}(x)-\eta^{-1}(x)\right), h(\mathrm{v})\right) \\
& =\mathbb{B}\left(\gamma\left(\eta^{-1}(x)\right)-\eta^{-1}(x), \mathrm{v}\right)=\tilde{\alpha}(\gamma)(\mathrm{v}) .
\end{aligned}
$$


Finally, for statement (4) we note that $F(g)=F\left(g^{n}\right)$ as sets, but the orientation on $F\left(g^{n}\right)$ is reversed if $n<0$. Again, choose $x \in \mathbb{A}^{2,1}$ and let $\mathrm{v} \in F(g)$. Then

$$
\begin{aligned}
\tilde{\alpha}\left(\gamma^{n}\right)(\mathrm{v}) & =\mathbb{B}\left(\gamma^{n}(x)-x, \mathrm{v}\right) \\
& = \begin{cases}\sum_{i=1}^{n} \mathbb{B}\left(\gamma^{i}(x)-\gamma^{(i-1)}(x), \mathrm{v}\right) & \text { if } n>0, \\
\sum_{i=1}^{|n|} \mathbb{B}\left(\gamma^{-i}(x)-\gamma^{(-i+1)}(x), \mathrm{v}\right) & \text { if } n<0,\end{cases} \\
& = \begin{cases}\sum_{i=1}^{n} \mathbb{B}\left(\gamma\left(\gamma^{(i-1)}(x)\right)-\gamma^{(i-1)}(x), \mathrm{v}\right) & \text { if } n>0, \\
\sum_{i=1}^{|n|} \mathbb{B}\left(\gamma^{-i}(x)-\gamma\left(\gamma^{-i}(x)\right), \mathrm{v}\right) & \text { if } n<0,\end{cases} \\
& = \begin{cases}\sum_{i=1}^{n} \tilde{\alpha}(g)(\mathrm{v}) & \text { if } n>0, \\
\sum_{i=1}^{|n|}-\tilde{\alpha}(g)(\mathrm{v}) & \text { if } n<0,\end{cases} \\
& =n \tilde{\alpha}(\gamma)(\mathrm{v}) .
\end{aligned}
$$

The sign of $\gamma$ is defined to be the sign of $\tilde{\alpha}(\gamma)(\mathrm{v})$, where $\mathrm{v}$ is any positive vector in $F(g)$ (see Definition [3.1). Since the orientation of $F\left(g^{n}\right)$ is reversed for $n<$ 0 , the sign of $\gamma$ and $\gamma^{-1}$ are the same. Furthermore, if $g$ is hyperbolic, then $\mathrm{x}^{0}\left(g^{n}\right)= \pm \mathrm{x}^{0}(g)$ is a positive vector in $F\left(g^{n}\right)$, so the sign of $\alpha\left(g^{n}\right)$ and the sign of $\tilde{\alpha}\left(g^{n}\right)\left(\mathrm{x}^{0}\left(g^{n}\right)\right)$ are the same.

We note here that the definition of $\alpha$ can be extended to most elliptic transformations, i.e. transformations whose linear parts have complex eigenvalues and are Lorentzian rotations about an axis. However, many of the results in Lemma 3.2 would not apply. One of most basic problems in extending the definition to elliptic transformations comes from defining the orientation on $F\left(g^{n}\right)$. There is no proper choice of orientation when $g$ is a rotation of order 2. If $g$ is an elliptic element not of order 2, then the orientation on $F\left(g^{n}\right)$ will switch back and forth as $n$ increases. In this case, the "sign" of an elliptic transformation would change as the powers change. It is interesting to note that statement (2) of Lemma 3.2 still holds for elliptic transformations.

\section{The OpPosite SIGN LEMMA}

We now prove Margulis' opposite sign lemma for parabolic and hyperbolic transformations.

Theorem 4.1. Let $\psi, \gamma \in \mathcal{H} \cup \mathcal{P}$. If the signs of $\psi$ and $\gamma$ are opposite, then $\langle\psi, \gamma\rangle$ does not act properly discontinuously on $\mathbb{A}^{2,1}$.

Proof. Lemma 2.5 deals with the case where $\psi$ and $\gamma$ are both hyperbolic. Furthermore, if both $\psi$ and $\gamma$ are parabolic, then there exists an integer $m$ such that $\psi^{m} \gamma$ is hyperbolic. The sign of $\psi^{m} \gamma$, assuming that it is not 0 , is opposite to either that of $\psi$ or $\gamma$.

Thus, to prove Theorem 4.1 it suffices to suppose that $\psi$ is parabolic, with $\tilde{\alpha}(\psi)$ negative, and $\gamma$ is hyperbolic, with $\tilde{\alpha}(\gamma)$ positive.

We will show that for a sufficiently large integer $n$, the transformation $\psi^{n} \gamma$ is hyperbolic and has a negative sign. Then we can invoke Lemma 2.5 to show that $\langle\psi, \gamma\rangle$ does not act properly discontinuously on $\mathbb{A}^{2,1}$. 
Substituting $\psi^{k} \gamma$ for $\gamma$ if necessary, for a large enough value of $k$, we may assume without loss of generality that $\psi^{n} \gamma$ is hyperbolic for all $n \geq 0$.

It will often prove useful to conjugate $\langle\psi, \gamma\rangle$ by some element of $\mathrm{SO}(2,1)^{0}$. By Lemma 2.2 (3), conjugating the group does not change the values of $\alpha$.

Set $p, g$, to be the linear parts of $\psi, \gamma$, respectively. Fix $x_{o} \in \mathbb{A}^{2,1}$ and set

$$
\begin{aligned}
& \mathbf{v}_{\psi}=\psi\left(x_{o}\right)-x_{o}, \\
& \mathbf{v}_{\gamma}=\gamma\left(x_{o}\right)-x_{o},
\end{aligned}
$$

so that we may think of $\mathbf{v}_{\psi}$ and $\mathrm{v}_{\gamma}$ as the translational parts of $\psi$ and $\gamma$, when $x_{o}$ plays the role of the origin. Observe that for any $n>0$,

$$
\psi^{n} \gamma\left(x_{o}\right)-x_{o}=\sum_{i=0}^{n-1} p^{i}\left(\mathbf{v}_{\psi}\right)+p^{n} \mathbf{v}_{\gamma} .
$$

Thus

$$
\begin{aligned}
\alpha\left(\psi^{n} \gamma\right) & =\sum_{i=0}^{n-1} \mathbb{B}\left(p^{i}\left(\mathbf{v}_{\psi}\right), x^{0}\left(p^{n} g\right)\right)+\mathbb{B}\left(p^{n} \mathbf{v}_{\gamma}, x^{0}\left(p^{n} g\right)\right) \\
& =\sum_{i=0}^{n-1} \mathbb{B}\left(\mathbf{v}_{\psi}, x^{0}\left(p^{n-i} g p^{i}\right)\right)+\mathbb{B}\left(p^{n} \mathbf{v}_{\gamma}, x^{0}\left(p^{n} g\right)\right)
\end{aligned}
$$

Finally, $\mathrm{x}^{0}(p)$ denotes the unique positive 1-eigenvector of $p$ of Euclidean length 1. In particular, $\mathbb{B}\left(\mathrm{v}_{\psi}, \mathrm{x}^{0}(p)\right)$ is negative.

The proof that $\alpha\left(\psi^{n} \gamma\right)$ is negative, for large enough $n$, is broken into three steps.

Step 1: Bound the term $\mathbb{B}\left(p^{n} \mathrm{v}_{\gamma}, \mathrm{x}^{0}\left(p^{n} g\right)\right)$ in (4). We may assume that $\mathrm{v}_{\gamma}$ is a scalar multiple of $x^{0}(p)$. Otherwise, we conjugate the group by an appropriate translation. Indeed, if $\tau$ denotes translation by $\mathrm{t}$, then

$$
\tau \gamma \tau^{-1}(x)-x=\mathrm{v}_{\gamma}-g(\mathrm{t})+\mathrm{t},
$$

so we need only find $\mathrm{t}$ such that $\mathrm{v}_{\gamma}-g(\mathrm{t})+\mathrm{t}$ is parallel to $\mathrm{x}^{0}(p)$. But since $\alpha(\gamma) \neq 0$, $\left\{\mathrm{v}_{\gamma}, \mathrm{x}^{-}(g), \mathrm{x}^{+}(g)\right\}$ forms a basis for $\mathbb{R}^{3}$, so that some scalar multiple of $\mathrm{x}^{0}(p)$ can be written as $\mathrm{v}_{\gamma}+\mathrm{x}$, where $\mathrm{x} \in\left\langle\mathrm{x}^{-}(g), \mathrm{x}^{+}(g)\right\rangle$. Since the range of $I d-g$ is precisely $\left\langle\mathrm{x}^{-}(g), \mathrm{x}^{+}(g)\right\rangle$, a suitable solution $\mathrm{t}$ can be found. Thus $p^{n} \mathrm{v}_{\gamma}=\mathrm{v}_{\gamma}$ for every $n>0$.

Now consider $p^{n} g$, the linear part of $\psi^{n} \gamma$. The fixed vector $\mathrm{x}^{+}\left(p^{n} g\right)$ converges to $\mathrm{x}^{0}(p)$ as $n \rightarrow \infty$. Similarly,

$$
\mathrm{x}^{-}\left(p^{n} g\right) \rightarrow \frac{g \mathrm{x}^{0}(p)}{\left\|g \mathrm{x}^{0}(p)\right\|}
$$

as $n \rightarrow \infty$. Since $\langle g, h\rangle$ is a free group, $g$ and $h$ have no common eigenvectors and $\rho\left(\mathrm{x}^{0}(p), \mathrm{x}^{0}\left(g p g^{-1}\right)\right)>0$ (recall that $\rho$ denotes Euclidean distance).

We can choose $\epsilon=\frac{1}{2} \rho\left(\mathrm{x}^{0}(p), \mathrm{x}^{0}\left(g p g^{-1}\right)\right)$, so that for sufficiently large $n, p^{n} g$ is $\epsilon$-hyperbolic. It follows that $\left\|\mathrm{x}^{0}\left(p^{n} g\right)\right\|$, thus $\left|\mathbb{B}\left(\mathrm{v}_{\gamma}, \mathrm{x}^{0}\left(p^{n} g\right)\right)\right|$, is bounded.

Step 2: Bound the terms in equation (4) of the form $\mathbb{B}\left(\mathrm{v}_{\psi}, \mathrm{x}^{0}\left(p^{n-i} g p^{i}\right)\right)$ by a negative number, for sufficiently large powers of $p$. Note that as $k$ and $i$ get large, $p^{k} g p^{i}$ is not $\epsilon$-hyperbolic for any chosen $\epsilon$. In fact, $\mathrm{x}^{ \pm}\left(p^{k} g p^{i}\right)$ both approach $\mathrm{x}^{0}(p)$.

Lemma 4.2. There exists $C_{-}<0$ and $M>0$ such that when both $i>M$ and $k>M$,

$$
\mathbb{B}\left(\mathrm{v}_{\psi}, \mathrm{x}^{0}\left(p^{k} g p^{i}\right)\right)<C_{-} .
$$


Proof. Conjugating the group if necessary, assume that

$$
\mathrm{x}^{0}(p)=\frac{\sqrt{2}}{2}\left[\begin{array}{l}
1 \\
0 \\
1
\end{array}\right] \text {. }
$$

The condition that the sign of $\psi$ is negative translates to the condition $v_{\psi}^{1}-v_{\psi}^{3}<0$, where $\mathbf{v}_{\psi}^{i}$ denotes the $i$ th coordinate of $\mathbf{v}_{\psi}$. We may further assume that $\mathbf{v}_{\psi}^{2}=0$, by conjugating the group again by a parabolic element in $\operatorname{SO}(2,1)^{0}$ that fixes $\mathrm{x}^{0}(p)$ (this is possible because $\left.\mathbb{B}\left(x^{0}(p), v_{\psi}\right) \neq 0\right)$. Set

$$
\begin{aligned}
& \mathrm{x}_{m}(\theta)=\frac{\sqrt{2}}{2}\left[\begin{array}{c}
\cos \theta \\
\sin \theta \\
1
\end{array}\right], \\
& \mathrm{x}_{p}(\theta)=\frac{\sqrt{2}}{2}\left[\begin{array}{c}
\cos \theta \\
-\sin \theta \\
1
\end{array}\right],
\end{aligned}
$$

where $\theta<\theta_{0}$, with $\theta_{0}>0$ small enough so that

$$
\mathrm{v}_{\psi}^{1}-\cos \theta_{0} \mathbf{v}_{\psi}^{3}<0
$$

We note that $\rho\left(\mathrm{x}_{m}\left(\theta_{0}\right), \mathrm{x}^{0}(p)\right)=\rho\left(\mathrm{x}_{m}\left(\theta_{0}\right), \mathrm{x}^{0}(p)\right)=2-2 \cos \theta_{0}$. Choose $M$ large enough so that for all $i, k>M, \rho\left(\mathrm{x}^{ \pm}\left(p^{k} g p^{i}\right), \mathrm{x}^{0}(p)\right)<2-2 \cos \theta_{0}$. Then we can write

$$
\begin{aligned}
& \mathrm{x}^{-}\left(p^{k} g p^{i}\right)=\mathrm{x}_{m}\left(\theta_{1}\right), \\
& \mathrm{x}^{+}\left(p^{k} g p^{i}\right)=\mathrm{x}_{p}\left(\theta_{2}\right),
\end{aligned}
$$

where $\theta_{1}, \theta_{2}<\theta_{0}$. Then

$$
\begin{aligned}
\mathbb{B}\left(\mathrm{v}_{\psi}, \mathrm{x}^{0}\left(p^{k} g p^{i}\right)\right) & =\frac{1}{\left|\mathbb{B}\left(\mathrm{x}_{m}\left(\theta_{1}\right), \mathrm{x}_{p}\left(\theta_{2}\right)\right)\right|} \mathbb{B}\left(\mathrm{v}_{\psi}, \mathrm{x}_{m}\left(\theta_{1}\right) \otimes \mathrm{x}_{p}\left(\theta_{2}\right)\right) \\
& =\frac{\left(\mathrm{v}_{\psi}^{1}-\cos \theta_{1} \mathrm{v}_{\psi}{ }^{3}\right) \sin \theta_{2}+\left(\mathrm{v}_{\psi}{ }^{1}-\cos \theta_{2} \mathrm{v}^{3}\right) \sin \theta_{1}}{1-\cos \left(\theta_{1}+\theta_{2}\right)},
\end{aligned}
$$

which decreases without bound. This proves our claim.

Step 3: Bound the sum of the remaining terms in equation (4). These are of the form $\mathbb{B}\left(\mathrm{v}_{\psi}, \mathrm{x}^{0}\left(p^{k} g p^{i}\right)\right)$, where either $i$ or $k$ is less than $M$. But then there is some fixed $\varepsilon>0$ such that each of these words is $\varepsilon$-hyperbolic. By Lemma 2.4, we can find a constant $C_{\varepsilon}$ such that $\mathbb{B}\left(\mathrm{v}_{\psi}, \mathrm{x}^{0}\left(p^{k} g p^{i}\right)\right)<C_{\varepsilon}$.

The number of such terms is less than $2 M$, which does not depend upon the number $n$. Therefore, we can choose a large enough $n$ so that the number of terms less than $C_{-}$increases, while the sum of the remaining terms is bounded from above by a number not depending on $n$.

\section{REFERENCES}

[1] Drumm, T., Fundamental polyhedra for Margulis space-times, Topology 31 (4) (1992), 677683. MR1191372 (94a:57051)

[2] _ Examples of nonproper affine actions, Mich. Math. J. 39 (1992), 435-442. MR 1182499 $(93 \mathrm{j}: 57022)$

[3] Drumm, T. and Goldman, W., The geometry of crooked planes, Topology 38, No. 2, (1999) 323-351. MR1660333 (99m:57034) 
[4] Fried, D. and Goldman, W., Three-dimensional affine crystallographic groups, Adv. Math. 47 (1983), 1-49. MR0689763 (84d:20047)

[5] Margulis, G., Free properly discontinuous groups of affine transformations, Dokl. Akad. Nauk SSSR 272 (1983), 937-940. MR0722330|(85e:22015)

[6] Complete affine locally flat manifolds with a free fundamental group, J. Soviet Math. 134 (1987), 129-134. MR0741860 (86h:22019)

[7] Mess, G., Lorentz spacetimes of constant curvature, preprint.

[8] Milnor, J., On fundamental groups of complete affinely flat manifolds, Adv. Math. 25 (1977), 178-187. MR0454886 (56:13130)

Department of Mathematics and Statistics, McMaster University, Hamilton, OnTARIO, CANADA L8S $4 \mathrm{~L} 7$

E-mail address: charette@math.mcmaster.ca

Department of Mathematics and Statistics, Swarthmore College, Swarthmore, PennSYLVANIA 19081

E-mail address: tad@swarthmore.edu

Current address: Department of Mathematics, University of Pennsylvania, Philadelphia, Pennsylvania 19104-6395

E-mail address: tad@math.upenn.edu 